\title{
THE
}

\section{Interdimensional degeneracies in van der Waals clusters and quantum Monte Carlo computation of rovibrational states}

\author{
M. P. Nightingale \\ University of Rhode Island \\ Mervlyn Moodley
}

Follow this and additional works at: https://digitalcommons.uri.edu/phys_facpubs

Terms of Use

All rights reserved under copyright.

\section{Citation/Publisher Attribution}

Nightingale, M. P., \& Moodley, M. (2005). Interdimensional degeneracies in van der Waals clusters and quantum Monte Carlo computation of rovibrational states. The Journal of Chemical Physics, 123, 04304. doi: 10.1063/1.1941107

Available at: https://doi.org/10.1063/1.1941107

This Article is brought to you for free and open access by the Physics at DigitalCommons@URI. It has been accepted for inclusion in Physics Faculty Publications by an authorized administrator of DigitalCommons@URI. For more information, please contact digitalcommons-group@uri.edu. 
Interdimensional degeneracies in van der Waals clusters and quantum Monte Carlo computation of rovibrational states

M. P. Nightingale, and Mervlyn Moodley

Citation: The Journal of Chemical Physics 123, 014304 (2005); doi: 10.1063/1.1941107

View online: https://doi.org/10.1063/1.1941107

View Table of Contents: http://aip.scitation.org/toc/jcp/123/1

Published by the American Institute of Physics

PHYSICS TODAY

WHITEPAPERS
ADVANCED LIGHT CURE ADHESIVES

\section{READ NOW}

Take a closer look at what these environmentally friendly adhesive systems can do
PRESENTED BY (8) MASTERBOND' 


\title{
Interdimensional degeneracies in van der Waals clusters and quantum Monte Carlo computation of rovibrational states
}

\author{
M. P. Nightingale ${ }^{a)}$ \\ Department of Physics, University of Rhode Island, Kingston, Rhode Island 02881 \\ Mervlyn Moodley \\ School of Physics, Howard College, University of KwaZulu-Natal, Durban, 4041, South Africa
}

(Received 13 April 2005; accepted 3 May 2005; published online 8 July 2005)

\begin{abstract}
Quantum Monte Carlo estimates of the spectrum of rotationally invariant states of noble gas clusters suggest interdimensional degeneracy in $N-1$ and $N+1$ spatial dimensions. We derive this property by mapping the Schrödinger eigenvalue problem onto an eigenvalue equation in which $D$ appears as a continuous variable. We discuss implications for quantum Monte Carlo and dimensional scaling methods. () 2005 American Institute of Physics. [DOI: 10.1063/1.1941107]
\end{abstract}

\section{INTRODUCTION}

One of the advantages of Monte Carlo methods is that they scale well with the number of degrees of freedom of a physical system. In this paper we consider van der Waals clusters consisting of $N$ bosonic Lennard-Jones atoms in $D$ spatial dimensions. We treat the atoms as "elementary" particles without internal degrees of freedom, so that in total we deal with clusters with $N D$ quantum-mechanical degrees of freedom. We are mainly interested in the energy spectra of these clusters.

Quantum Monte Carlo computations can be made much more efficient by the use of optimized trial wave functions, as is well known. With currently available methods, as a matter of fact, the problem of computing rovibrational spectra with Monte Carlo methods is virtually intractable without good trial functions. One of the questions of interest is the relative importance of the quality of these trial wave functions for $n$-body correlations with $n$ in excess of the commonly used correlations with $n=2$ and $n=3$. In this context, the idea of varying the spatial dimensionality of the system quite naturally suggests itself, because particles can be more compact in higher dimensions, which suggests that correlations involving a higher number of particles might become more important as the spatial dimensionality increases. While we have not found clear numerical evidence to support this idea, ${ }^{1}$ our computations did produce an interesting by-product, which forms the topic of this paper.

Our computations showed that the energy spectra of $N$ particles in $N-1$ and $N+1$ spatial dimensions are numerically indistinguishable for states invariant under rotation and translation. ${ }^{1}$ Indeed, in this paper we show that for these $S$ states and for $D \geqslant N-1$, the $N$-particle time-independent Schrödinger equation can be transformed into an eigenvalue equation involving a differential operator with $\frac{1}{2}(N-1)$ $N$-independent variables and an effective potential in which the spatial dimension $D$ appears as a continuously varying parameter. This effective potential turns out to depend qua-

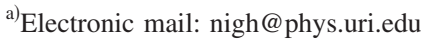

dratically on $D$ and is symmetric about $D=N$. This implies the aforementioned interdimensional degeneracy, in addition to a relationship between the wave functions for $D=N-1$ and $D=N+1$ dimensions. We note here that, as is also manifest in the frustration of the classical system for $D<N-1$-frustration, for example, in the sense that not all interatomic distances can be equal-the spectrum for values of $D<N-1$ cannot be obtained by analytic continuation of the spectrum for $D \geqslant N-1$; we shall return to this in the discussion at the end of this paper.

The interdimensional degeneracy was also derived recently by $\mathrm{Gu} e t a l .{ }^{2}$ by a group-theoretical method. To the best of our knowledge, interdimensional degeneracies of $S$ states of Lennard-Jones clusters have not been observed before, with the exception of the two-body cluster in one and three dimensions which follows from the standard separation of variable solution of the Schrödinger equation.

Even though an extension of our study to dimensions higher than the physical three dimensions is primarily of academic interest, the effect of spatial dimension on quantum systems has been studied since the early days of quantum physics. In fact, Fock ${ }^{3}$ as early as 1935 showed that there exists a relationship between the hydrogenlike wave functions and four-dimensional hyperspherical harmonics. ${ }^{4}$ The hyperspherical coordinate method was used in the late 1970s to discover interdimensional degeneracies in electron systems. For the one-electron system a transformation was found that reveals interdimensional degeneracy between a system in $D$ dimension and angular momentum $l$ with the same system in $D \pm 2$ dimensions and angular momentum $l \mp 1 .^{5}$ Many-electron systems have also been shown to exhibit interdimensional degeneracies. ${ }^{6}$ Further references to other interdimensional studies can be found in Ref. 2.

The structure of this paper is as follows. In Sec. II A we briefly summarize the quantum Monte Carlo technique for excited states developed in Refs. 7 and 8 to obtain optimized trial wave functions for van der Waals clusters. In this method, trial wave functions are developed that can be improved systematically. These trial wave functions are linear combinations of elementary basis functions with nonlinear 
variational parameters. The elementary basis functions consist of a prefactor and exponential polynomial that is formulated in terms of all possible $N$-body correlations. In Sec. II B we present Monte Carlo energy estimates obtained for selected few-body van der Waals clusters in a limited number of dimensions ranging from $D=1$ to $D=6$. In this study we consider van der Waal clusters composed of atoms of $\mathrm{Kr}$, Ar, $\mathrm{Ne}$, and the hypothetical $\frac{1}{2}-\mathrm{Ne}$, which has half the (dimensionless) mass of $\mathrm{Ne}$. $\mathrm{Kr}$ can be considered as a semiclassical case while that of $\frac{1}{2}-\mathrm{Ne}$ is more quantum mechanical in nature. Section III is devoted to the exact derivation of dimensional degeneracy, with some of the results postponed to the Appendix. In Sec. IV we discuss the relevance of our results, in particular, for dimensional scaling methods.

\section{MONTE CARLO APPROACH}

\section{A. Optimization of ground- and excited-state wave functions}

We consider clusters in $D$ dimensions consisting of $N$ atoms with positions specified by the $D \times N$ matrix of Cartesian coordinates $\mathbf{R}=\left(\mathbf{r}_{1} \mathbf{r}_{2} \cdots \mathbf{r}_{N}\right)$, with

$$
\mathbf{r}_{i}=\left(\begin{array}{c}
x_{1 i} \\
\vdots \\
x_{D i}
\end{array}\right)
$$

We shall use the following definitions:

$$
\begin{aligned}
& \mathbf{r}_{i j}=\mathbf{r}_{j}-\mathbf{r}_{i}, \\
& r_{i j}=\left|\mathbf{r}_{i j}\right|,
\end{aligned}
$$

for difference vectors and their lengths.

For a system of $N$ bosonic van der Waals atoms with atomic mass $\mu$ and interacting via a pair potential, the dimensionless Hamiltonian is

$$
H=-\frac{1}{2 m} \sum_{i=1}^{N} \nabla_{i}^{2}+\sum_{(i, j)} V\left(r_{i j}\right),
$$

with

$$
\nabla_{i}^{2}=\sum_{\alpha=1}^{D} \frac{\partial^{2}}{\partial x_{\alpha i}^{2}}
$$

and where $V$ is the dimensionless Lennard-Jones potential,

$$
V(r)=\frac{1}{r^{12}}-\frac{2}{r^{6}} .
$$

The inverse dimensionless mass is given by $m^{-1}$ $=\hbar^{2} / 2^{1 / 3} \mu \sigma^{2} \epsilon$, which is proportional to the square of the de Boer parameter, ${ }^{9}$ where $\epsilon$ is the minimum of the LennardJones potential and $2^{1 / 6} \sigma$ the corresponding interparticle distance.

A preliminary step in our optimization procedure is to generate a sample of configurations $\mathbf{R}_{\sigma}$, with $\sigma=1, \ldots, s$, which are sampled from a relative probability density function $\psi_{g}\left(\mathbf{R}_{\sigma}\right)^{2}$. The guiding function $\psi_{g}$ used for the computations reported in this paper is defined in terms of a trial function $\widetilde{\psi}$, which approximates the ground state. In simple cases, we used $\psi_{g}^{2}=\widetilde{\psi}^{2 / \rho}$, where the parameter $\rho$ is chosen in the range of $2 \lesssim \rho \lesssim 3$. Where necessary, we used a more sophisticated guiding function ${ }^{10}$ so as to generate a sample with substantial overlap with all the excited states under consideration.

The trial wave functions are linear combinations of elementary basis functions $\beta_{i}$, each of which implicitly depends on nonlinear variational parameters, and we use different procedures to optimize the linear and nonlinear parameters. For reasons explained in detail below, we define the reweighted functions $\hat{\beta}_{i}(R)=\psi_{g}(R)^{-1} \beta_{i}(R)$ and $\hat{\beta}_{i}^{\prime}(R)$ $=\psi_{g}(R)^{-1} H \beta_{i}(R)$. For a complete set of elementary basis functions $\beta_{i}$, the Schrödinger equation can be written in the form

$$
\hat{\beta}_{i}^{\prime}\left(\mathbf{R}_{\sigma}\right)=\sum_{j=1}^{n} \hat{\beta}_{j}\left(\mathbf{R}_{\sigma}\right) \mathcal{E}_{j i} .
$$

In practical applications, the set of functions $\beta_{i}$ is, of course, far from complete, but the $n \times n$ matrix $\mathcal{E}$ may still be determined by solving Eq. (6) for $\mathcal{E}$ in a least-squares sense given the reweighting just introduced. Note that Eq. (6) is exactly satisfied if the functions $\beta_{i}$ span an invariant subspace of the Hamiltonian $H$, even if they do not form a complete set; this provides an important zero-variance principle for the corresponding part energy spectrum.

It is convenient to rewrite Eq. (6) in matrix form

$$
\mathbf{B}^{\prime}=\mathbf{B} \mathcal{E},
$$

where $B_{\sigma i}=\hat{\beta}_{i}\left(R_{\sigma}\right)$ and $B_{\sigma i}^{\prime}=\hat{\beta}_{i}^{\prime}\left(R_{\sigma}\right)$. Multiplying Eq. (7) from the left by the transpose of $\mathbf{B}$, one obtains by inversion

$$
\mathcal{E}=\left(\mathbf{B}^{T} \mathbf{B}\right)^{-1}\left(\mathbf{B}^{T} \mathbf{B}^{\prime}\right) \equiv \hat{\mathbf{N}}^{-1} \hat{\mathbf{H}}
$$

with $\hat{N}_{i j}=\Sigma_{\sigma} \hat{\beta}_{i}\left(R_{\sigma}\right) \hat{\beta}_{j}\left(R_{\sigma}\right)$ and $\hat{H}_{i j}=\Sigma_{\sigma} \hat{\beta}_{i}\left(R_{\sigma}\right) \hat{\beta}_{j}^{\prime}\left(R_{\sigma}\right)$. As can be easily verified, Eq. (8) is indeed the least-squares solution of Eq. (6). Note that for an infinite sample the hermiticity of the Hamiltonian guarantees that $\hat{\mathbf{H}}$ is a symmetric matrix, but this is not the case for a finite Monte Carlo sample. If $\hat{\mathbf{H}}$ is symmetrized in Eq. (8), the resulting $\mathcal{E}$ no longer satisfies the least-squares property nor the aforementioned zero-variance principle.

The optimal linear combinations of the basis functions $\beta_{i}$ are computed by constructing the spectral decomposition of $\mathcal{E}$.

$$
\mathcal{E}_{i j}=\sum_{k=1}^{n} d_{i}^{k} \widetilde{E}_{k} \hat{d}_{j}^{k},
$$

where $\hat{d}_{j}^{k}$ and $d_{i}^{k}$ are the components of the left and right eigenvectors of $\mathcal{E}$ with eigenvalues $\widetilde{E}_{k}$. This yields the trial functions

$$
\widetilde{\psi}^{k}=\sum_{i=1}^{n} d_{i}^{k} \beta_{i} .
$$

Before we continue this review of our optimization procedure, some comments should be made. First of all, the matrix $\hat{\mathbf{N}}$ frequently is ill conditioned. This problem can be 
TABLE I. Ground-state energies $E_{1}$ (with errors in the least significant digit) and deviations from quadratic fits $\Delta E_{1}$ for $\mathrm{Kr}_{3}, \mathrm{Ar}_{3}$, and $\frac{1}{2}-\mathrm{Ne}_{3}$ in dimensions $D=1$ through $D=6$.

\begin{tabular}{|c|c|c|c|c|c|c|}
\hline \multirow[b]{2}{*}{$D$} & \multicolumn{2}{|c|}{$\mathrm{Kr}_{3}$} & \multicolumn{2}{|c|}{$\mathrm{Ar}_{3}$} & \multicolumn{2}{|c|}{$\frac{1}{2}-\mathrm{Ne}_{3}$} \\
\hline & $E_{1}$ & $\Delta E_{1}$ & $E_{1}$ & $\Delta E_{1}$ & $E_{1}$ & $\Delta E_{1}$ \\
\hline 1 & -1.8725485476 & $-9 \times 10^{-1}$ & -1.73480871 & $-8 \times 10^{-1}$ & -0.895584 & $-4 \times 10^{-1}$ \\
\hline 2 & -2.7604613515 & $2 \times 10^{-10}$ & -2.55295322 & $-1 \times 10^{-9}$ & -1.302484 & $-7 \times 10^{-7}$ \\
\hline 3 & -2.7605552787 & $6 \times 10^{-10}$ & -2.55328943 & $1 \times 10^{-8}$ & -1.308442 & $9 \times 10^{-6}$ \\
\hline 4 & -2.7604613513 & $-5 \times 10^{-11}$ & -2.55295322 & $-1 \times 10^{-9}$ & -1.302483 & $-2 \times 10^{-6}$ \\
\hline 5 & -2.7601795698 & $-1 \times 10^{-9}$ & -2.55194461 & $-2 \times 10^{-8}$ & -1.284627 & $-1 \times 10^{-5}$ \\
\hline 6 & -2.7597099376 & $5 \times 10^{-10}$ & -2.55026364 & $7 \times 10^{-9}$ & -1.254901 & $5 \times 10^{-6}$ \\
\hline
\end{tabular}

dealt with by using a singular value decomposition of the matrix $\mathbf{B}$ to obtain a numerically regularized inverse of $\hat{\mathbf{N}}^{7,8}$ Secondly, we mention that Eq. (10) can also be derived from the condition that the quantum-mechanical expectation value of the Hamiltonian in the states $\widetilde{\psi}^{k}$ is stationary with respect to variation of the coefficients $d_{i}^{k}$. This condition yields a generalized eigenvalue equation involving matrices $\mathbf{N}$ and $\mathbf{H}$, the Monte Carlo estimators of which are the matrices $\hat{\mathbf{N}}$ and $\hat{\mathbf{H}}$ introduced previously. The reweighting defined before Eq. (6) was introduced so that these estimators are unbiased.

As mentioned, the basis functions $\beta_{i}$ depend implicitly on nonlinear variational parameters. These are optimized iteratively and it should be kept in mind that for each choice of the nonlinear parameters, new optimized linear parameters have to be computed. The full optimization of all parameters therefore entails a linear optimization nested in a nonlinear one. The linear optimization is a standard linear algebra problem; the optimization of the nonlinear parameters is performed by minimizing the variance of the local energy of the wave function:

$$
\chi^{2}=\frac{\sum_{\sigma=1}^{s}\left[\hat{\psi}^{k \prime}\left(\mathbf{R}_{\sigma}\right)-\widetilde{E}_{k} \hat{\psi}^{k}\left(\mathbf{R}_{\sigma}\right)\right]^{2}}{\sum_{\sigma=1}^{s} \hat{\psi}^{k}\left(\mathbf{R}_{\sigma}\right)^{2}},
$$

where $\hat{\psi}^{k}=\psi_{g}^{-1} \widetilde{\psi}^{k}$ and $\hat{\psi}^{k \prime}=\psi_{g}^{-1} H \widetilde{\psi^{k}}$.

The trial wave functions produced by this method yield estimates of the energy levels that are upper bounds to the exact energies, if statistical errors are negligible. To reduce the resulting systematic errors, the so-called variational errors, we employ these optimized wave functions as the basis functions in a correlation function Monte Carlo calculation. ${ }^{11-13}$ This reduces the variational bias in the eigenvalue estimates, but it usually increases the statistical errors in the estimates. In a formal sense, the reduction of variational errors obtained in correlation function Monte Carlo is accomplished by introducing a new and improved basis by means of the substitution,

$$
\beta_{i}(R) \rightarrow \exp (-t H) \beta_{i}(R) \equiv \beta_{i}(R, t) .
$$

For increasing projection time $t$ the spectral weight of more highly excited states in the new basis is reduced, and with it the variational error. In the limit $t \rightarrow \infty$ all states of the new basis collapse onto the ground state, which implies that as $t$ increases, the overlap matrix of the $t$-dependent basis states becomes more nearly singular, which increases the statistical errors. In principle, the errors increase exponentially; in practice, the method as we currently use it, breaks down once the Monte Carlo estimate of the overlap matrix develops negative eigenvalues.

We use elementary basis functions of the following general form: ${ }^{14}$

$$
\beta_{i}(R)=s_{i}(R) \exp \left(\sum_{j} a_{j} s_{j}(R)+\sum_{\sigma<\tau} A\left(r_{\sigma \tau}\right)\right),
$$

where the term involving $A$ imposes short- and long-range asymptotics; the $s_{i}$ and $s_{j}$ are bosonically symmetrized monomials. The exact structure of these basis functions is of no concern in this paper. A detailed description of the abovementioned method and the structure of the basis functions can be found in Refs. 7 and 8 .

\section{B. Numerical results in various dimensions}

In this section we present numerical results that show that the energy spectrum as a function of dimensionality for $D \geqslant N-1$ is symmetric about $D=N$. We discuss results for $\mathrm{Kr}, \mathrm{Ar}, \mathrm{Ne}$, and the hypothetical $\frac{1}{2}-\mathrm{Ne}$, which are defined, respectively, by the following inverse masses: 1.9128 $\times 10^{-4}(\mathrm{Kr}), 6.9635 \times 10^{-4}(\mathrm{Ar}), 7.0920 \times 10^{-3}(\mathrm{Ne})$, and $1.4184 \times 10^{-2}\left(\frac{1}{2}-\mathrm{Ne}\right)$.

\section{The three-body case}

Table I shows the ground-state energies $E_{1}$ for $\mathrm{Kr}_{3}, \mathrm{Ar}_{3}$, and $\frac{1}{2}-\mathrm{Ne}_{3}$ in dimensions ranging from $D=1$ to $D=6$. We fitted the computed values for $D \geqslant 2$ to a parabola with its minimum at $D=2$. The difference between the computed and fitted results $\Delta E_{1}$ is also shown in Table I. As is the case with the classical minimum of the energy, which equals -2.03 for $D=1$ and -3 for $D \geqslant 2$, the quantum-mechanical groundstate energy at $D=1$ is nowhere near the curve.

\section{The four-body case}

Table II shows the ground-state energies $E_{1}$ for a fourbody cluster, $\mathrm{Ar}_{4}$, in various dimensions. From these results it is evident that an interdimensional degeneracy exists in $D=3$ and $D=5$ dimensions. Again, $\Delta E_{1}$ represents the difference of the computed energies and the results obtained from 
TABLE II. Ground-state energies (with errors in the least significant digit) and deviations from quadratic fits $\Delta E_{1}$ for $\mathrm{Ar}_{4}$ in dimensions $D=1$ through $D=6$.

\begin{tabular}{ccc}
\hline \hline$D$ & $E_{1}$ & $\Delta E_{1}$ \\
\hline 1 & -2.62562256 & $-2 \times 10^{-0}$ \\
2 & -4.32951795 & $-8 \times 10^{-1}$ \\
3 & -5.11814605 & $-2 \times 10^{-9}$ \\
4 & -5.11865384 & $3 \times 10^{-9}$ \\
5 & -5.11814605 & $-2 \times 10^{-9}$ \\
6 & -5.11662270 & $1 \times 10^{-9}$ \\
\hline \hline
\end{tabular}

a parabolic fit with its minimum at $D=N=4$, this time excluding $D=1$ and $D=2$. The quantum-mechanical estimates can be compared with the classical minima, viz., -3.07 for $D=1,-5.07$ for $D=2$, and -6 for $D \geqslant 3$.

\section{Excited states}

Thus far we have only numerically verified that interdimensional degeneracies exist for ground-state energies. Tables III and IV strongly suggest that the same holds for excited states. The first table shows the four lowest excitedstate energies obtained for $\mathrm{Ar}_{3}$ in $D=2,3$, and 4 dimensions. Once again, as observed for the ground-state energies, these degeneracies exist in this three-body cluster for the $D=2$ and $D=4$ case. The $D=3$ case is included in this table to indicate that the energies obtained here are different and lower than the other two cases. The second table shows the four lowest excited-state energies obtained for $\mathrm{Ar}_{4}$ in $D=3$ and 5 dimensions; we denote energy levels by $E_{1}<E_{2}<\cdots$.

The results in Table $\mathrm{V}$ illustrate the loss of accuracy that occurs for five-particle clusters. The differences between the estimates of the energies of corresponding levels for four and six dimensions are due to the failure to converge of the correlation function Monte Carlo. This reflects the fact that our trial wave functions can in principle be systematically improved only for cluster sizes $N \leqslant 4$, because they contain fully adjustable $n$-body correlations with $n \leqslant 4$ only.

\section{EXACT RESULTS}

\section{A. Clusters in arbitrary number of dimension}

The Schrödinger equation for an $N$-particle cluster in $D$ spatial dimensions is a differential equation in $N D$ variables. For an $S$ state the wave function is invariant under rotations and translations. Therefore, one can write the wave function as a function of fewer than $N D$ variables. To accomplish this we proceed as follows.

TABLE III. Comparison of the excited-state energies $E_{k}$ (with errors in the least significant digit) of $\mathrm{Ar}_{3}$ in $D=2,3$, and 4 dimensions.

\begin{tabular}{clll}
\hline \hline$k$ & \multicolumn{1}{c}{$D=2$} & \multicolumn{1}{c}{$D=3$} & \multicolumn{1}{c}{$D=4$} \\
\hline 2 & -2.2498602 & -2.2501855 & -2.249860 \\
3 & -2.1260388 & -2.126361 & -2.126039 \\
4 & -1.996153 & -1.99643 & -1.996153 \\
5 & -1.9463 & -1.9467 & -1.9463 \\
\hline \hline
\end{tabular}

TABLE IV. Comparison of the excited-state energies $E_{k}$ (with errors in the least significant digit) of $\mathrm{Ar}_{4}$ in $D=3$ and 5 dimensions.

\begin{tabular}{cll}
\hline \hline$k$ & \multicolumn{1}{c}{$D=3$} & \multicolumn{1}{c}{$D=5$} \\
\hline 2 & -4.80089773 & -4.80089775 \\
3 & -4.7251567 & -4.7251566 \\
4 & -4.630025 & -4.630025 \\
5 & -4.586389 & -4.586384 \\
\hline \hline
\end{tabular}

Consider the $N-1$ difference vectors $\mathbf{r}_{21}, \mathbf{r}_{31}, \ldots, \mathbf{r}_{N 1}$, as defined by Eq. (2a). Note that these vectors cannot be linearly independent unless $D \geqslant N-1$, in which case they define a parallelepiped $P$. Precisely $\frac{1}{2}(N-1) N$-independent variables are required to define $P$ up to a congruence transformation. One possible choice of such variables consists of (1) the angles $\theta_{i j}$ between the vectors $\mathbf{r}_{i 1}$ and $\mathbf{r}_{j 1}$ or their cosines,

$$
g_{i j}=\frac{\mathbf{r}_{1 i} \cdot \mathbf{r}_{1 j}}{r_{1 i} r_{1 j}},
$$

with $1<i<j \leqslant N$ and (2) the lengths of the vectors $\mathbf{r}_{i 1}$ with $1<i \leqslant N$. Alternatively, as independent variables one may choose the lengths of all distinct interparticle distances $r_{i j}$ $=r_{j i}$ with $i \neq j$. These are the variables we shall use in this paper with the assumption, required for linear independence, that $D \geqslant N-1$.

\section{B. Generalized Schrödinger equation}

We consider a $D$-dimensional Schrödinger equation of the form

$$
\left(-\sum_{i=1}^{N} \frac{1}{2 m_{i}} \nabla_{i}^{2}+V\right) \psi=E \psi,
$$

with a Hamiltonian slightly more general than the one defined in Eq. (3) with a potential that is rotationally and translationally invariant, but not necessarily a sum of two-body contributions. Furthermore, the mass of each particle may be different.

We restrict ourselves to $S$ states and to the cases in which $D \geqslant N-1$ so that, as discussed in Sec. III A, the wave functions can be considered to be a function of independent interparticle distances $r_{i j}$ with $1<i<j \leqslant N$.

By straightforward application of the differential operator identity,

TABLE V. Comparison of ground- and excited-state energies $E_{k}$ (with uncontrolled errors) of $\mathrm{Ne}_{5}$ in $D=4$ and 6 dimensions.

\begin{tabular}{lll}
\hline \hline$k$ & \multicolumn{1}{c}{$D=4$} & \multicolumn{1}{c}{$D=6$} \\
\hline 1 & -5.82121 & -5.82121 \\
2 & -5.3466 & -5.3372 \\
3 & -5.26 & -5.18 \\
4 & -5.06 & -4.99 \\
5 & -4.95 & -4.91 \\
\hline
\end{tabular}




$$
\frac{\partial}{\partial x_{\alpha i}}=\sum_{j \neq i} \frac{\partial r_{i j}}{\partial x_{\alpha i}} \frac{\partial}{\partial r_{i j}},
$$

one obtains

$$
\nabla_{i}^{2}=\sum_{j \neq i} a_{i ; j} \frac{\partial}{\partial r_{i j}}+\sum_{j, k \neq i} g_{i ; j k} \frac{\partial^{2}}{\partial r_{i j} \partial r_{i k}},
$$

where

$$
a_{i ; j}=\sum_{\alpha=1}^{D} \frac{\partial^{2} r_{i j}}{\partial x_{\alpha i}^{2}}=\frac{D-1}{r_{i j}},
$$

and

$$
g_{i ; j k}=\sum_{\alpha=1}^{D} \frac{\partial r_{i j}}{\partial x_{\alpha i}} \frac{\partial r_{i k}}{\partial x_{\alpha i}}=\frac{\mathbf{r}_{i j} \cdot \mathbf{r}_{i k}}{r_{i j} r_{i k}} .
$$

With the interparticle distances as independent variables, the Schrödinger equation assumes a form that involves (1) a linear differential operator that explicitly depends on the spatial dimensionality $D$, and (2) a second-order differential and a potential-energy operator that are independent of $D$, as is clear from Eqs. (17) and (19).

Next, we transform the Schrödinger equation into an equation in which the second-order operator is unchanged, the linear operator is absent, and in which the potential is modified by an additional term. ${ }^{15}$ This is accomplished as follows:

$$
\mathbf{H} \psi=E \psi \rightarrow \mathbf{H}^{\prime} \phi=E \phi,
$$

with

$$
\psi=\chi \phi
$$

and

$$
\mathbf{H}^{\prime}=\frac{1}{\chi} \mathbf{H} \chi .
$$

The action of the operator on the right-hand side of an arbitrary function is to be evaluated from right to left, so that multiplying by $\chi$ takes precedence over operating by $\mathbf{H}$.

This yields a special case of Eq. 3.8 of Ref. 16

$$
\mathbf{H}^{\prime}=V-\sum_{i=1} \frac{1}{2 m_{i}}\left(S_{i}+T_{i}+U_{i}\right),
$$

with

$$
\begin{aligned}
& S_{i}=\sum_{j, k \neq i} g_{i ; j k} \frac{\partial^{2}}{\partial r_{i j} \partial r_{i k}}, \\
& T_{i}=\sum_{j \neq i}\left(a_{i ; j}+2 \sum_{k \neq i} g_{i ; j k} \chi^{-1} \frac{\partial \chi}{\partial r_{i k}}\right) \frac{\partial}{\partial r_{i j}}, \\
& U_{i}=\sum_{j \neq i} a_{i ; j} \chi^{-1} \frac{\partial \chi}{\partial r_{i j}}+\sum_{j, k \neq i} g_{i ; j k} \chi^{-1} \frac{\partial^{2} \chi}{\partial r_{i k} \partial r_{i k}} .
\end{aligned}
$$

We define square matrices of order $N-1$,

$$
\hat{G}_{i}=\left(r_{i j} g_{i ; j k} r_{i k}\right)_{j, k \neq i},
$$

for $i=1, \ldots, N$. The matrix $\hat{G}_{i}$ is the Grammian associated with the $N-1$ vectors $\mathbf{r}_{i j}$ with $j=1, \ldots, i-1, i+1, \ldots, N$. Its determinant

$$
\omega=\operatorname{det}\left(\hat{G}_{i}\right)
$$

is the square of the volume of the parallelepiped defined by the vectors pointing from particle $i$ to all other particles. This volume is equal to $N$ ! times the volume of the $(N-1)$ simplex of which the $N$ particles are the vertices, which explains why $\omega$ does not depend on $i$, as our notation indicates.

In the Appendix we show that $T_{i}$ vanishes for the choice

$$
\chi=\omega^{(1-D) / 4} \text {, }
$$

while

$$
\begin{aligned}
& U_{i}=\frac{1}{8}\left[(N-1)^{2}-(N-D)^{2}\right] \sum_{j \neq i} \frac{1}{r_{i j}} \frac{\partial \log \omega}{\partial r_{i j}}, \\
& =\frac{(N-1)^{2}-(N-D)^{2}}{16 \omega^{2}} \sum_{j, k \neq i} \frac{\partial \omega}{\partial r_{i j}} g_{i ; j k} \frac{\partial \omega}{\partial r_{i k}} .
\end{aligned}
$$

Clearly, $U_{i}$ depends on the spatial dimensionality via its $D$-dependent amplitude, which is symmetric in $D$ about $D=N$. Recalling that this derivation is valid only for values for $D \geqslant N-1$, we find that for $S$ states the Schrödinger equation has the same energy eigenvalues in $D=N-1$ and $D=N$ +1 - and for those values of $D$ only-while the eigenstates are related via

$$
\frac{\psi(D=N-1)}{\chi(D=N-1)}=\frac{\psi(D=N+1)}{\chi(D=N+1)} .
$$

Furthermore, using the fact that $g_{i ; j k}$, defined in Eq. (19), is an inner product, one can rewrite the sum in Eq. (30b) as a sum of squares. This implies that to linear order in perturbation theory the energy eigenvalues depend quadratically on $D$ with a minimum at $D=N$, in agreement with our numerical estimates presented in Sec. II B.

\section{DISCUSSION}

We transformed the Schrödinger equation for the rotationally and translationally invariant states of an $\mathrm{N}$-particle cluster in $D \geqslant N-1$ spatial dimensions into a differential equation in $\frac{1}{2}(N-1) N$-independent variables in which the dependence on $D$ is fully contained in an effective potential energy. Here $D$ can be interpreted as a continuously varying parameter, as is commonly done in dimensional scaling studies. ${ }^{17}$ In agreement with work by Gu et al., ${ }^{2}$ we observed that there exists an interdimensional degeneracy of an $N$-body cluster in $D=N-1$ and $D=N+1$ dimensions. This degeneracy exists for all $S$ states, i.e., for both the ground and excited states. Furthermore, the minimum energy was observed to be at the dimension $D=N$.

We stumbled upon this interdimensional degeneracy numerically by Monte Carlo methods, for which the generalization to arbitrary discrete dimensions is simple. In retrospect, knowing that this degeneracy is an exact property of 
the Schrödinger equation is useful because it provides a powerful check of the validity of our computer code and of our estimates of systematic and statistical errors in our numerical results.

Our results have another interesting implication. Our transformation of the Schrödinger equation to a differential equation in $\frac{1}{2}(N-1) N$-independent variables is valid only for $D \geqslant N-1$. However, $D$ in the resulting equation can be interpreted as a continuous variable, and the equation has an analytical continuation for $D<N-1$ and is symmetric about $D$ $=N$. As a consequence, the energy spectrum for $D<N-1$ of the transformed equation is analytic in $D$ and symmetric about $D=N$. Whatever might be the meaning of this spectrum obtained by analytic continuation of the spectrum for large values of the $D$ spectrum, it cannot have anything to do with the physical spectrum of $N$-particle clusters for $D<N$ -1 . This in turn implies that there is a fundamental problem with the work by Gonzalez and Leal, ${ }^{18}$ who have used $1 / D$ expansion to estimate energy levels of Lennard-Jones clusters in $D=3$ with $N=3,4, \ldots, 7$, and 13 . If such calculations could be done to infinite order and resummed to yield a convergent expression valid for all $D$, the result would agree with the analytic continuation discussed above, but not with the physics of clusters with more than four particles in three dimensions.

\section{ACKNOWLEDGMENT}

This research was supported by the United States National Science Foundation under Grant No. ITR 0218858.

\section{APPENDIX: TECHNICAL DETAILS}

Without loss of generality we can restrict our discussion to the contribution to the transformed Hamiltonian $\mathbf{H}^{\prime}$ of the kinetic energy of particle $i=N$. Correspondingly, we shall simplify our notation as follows:

$$
\begin{aligned}
& a_{i}=a_{N ; i}, \\
& g_{i j}=g_{N ; i j}, \\
& r_{i}=r_{N i} .
\end{aligned}
$$

Note, in particular, that $r_{i}$ is not the distance of particle $i$ to the origin, as suggested by convention and Eq. (1), but rather the distance of particle $N$ to particle $i$.

Define

$$
\hat{G}=\left(r_{i} g_{i j} r_{j}\right)_{i, j=1}^{N-1} .
$$

Then the square of volume of the parallelepiped is given by the Grammian

$$
\omega=\operatorname{det}(\hat{G}) .
$$

Consider a symmetric $s \times s$ matrix $M$ of the form

$$
m_{i j}= \begin{cases}u_{i}\left(x_{i}\right), & \text { if } i=j, \\ v_{i j}\left(x_{i}, x_{j}\right)=v_{j i}\left(x_{j}, x_{i}\right), & \text { if } i<j .\end{cases}
$$

Since only row and column $i$ depend on $x_{i}$ this implies that $\frac{\partial \operatorname{det}(M)}{\partial x_{i}}=\sum_{j=1}^{N} M_{j i}\left(\frac{\partial u_{i}\left(x_{i}\right)}{\partial x_{i}} \delta_{i j}+2 \frac{\partial v_{i j}\left(x_{i}, x_{j}\right)}{\partial x_{i}}\left(1-\delta_{i j}\right)\right)$,

where $M_{i j}$ is the $(i, j)$ cofactor of $M$

Now

$$
\frac{\partial \hat{G}}{\partial r_{p}}=r_{p} C^{p}
$$

$i=1, \ldots, N-1$, where $C^{p}$ has elements equal to unity on the off-diagonal of row and column $p$ and equal for diagonal element $p$, i.e.,

$$
\begin{gathered}
c_{i j}^{(p)}=\delta_{i p}+\delta_{j p}, \\
\frac{\partial \omega}{\partial r_{i}}=2 r_{i} \sum_{j=1}^{N-1} \hat{G}_{j i} .
\end{gathered}
$$

From this we find

$$
\sum_{i} \hat{g}_{j i} \frac{\partial \omega}{\partial r_{i}} \frac{1}{r_{i}}=2 \omega
$$

This can be verified by solving this last equation for $\left(\partial \omega / \partial r_{i}\right) 1 / r_{i}$ by means of Cramer's rule, which indeed yields back Eq. (A10). Thus we find

$$
\sum_{i} g_{j i} \frac{\partial \omega}{\partial r_{i}}=2 \frac{\omega}{r_{j}}
$$

from which it follows that

$$
\chi=\omega^{(1-D) / 4},
$$

so that

$$
\sum_{i=1}^{N-1} g_{j i} \frac{\partial \chi}{\partial r_{i}}=\frac{1}{2}(1-D) \frac{\chi}{r_{j}}=-\frac{1}{2} \chi a_{j},
$$

which shows that the linear differential operators vanish in $T_{N}$.

To make the dependence on the spatial dimension $D$ explicit, we write the first term on the right-hand side of Eq. (26) as

$$
\sum_{j=1}^{N-1} a_{j} \chi^{-1} \frac{\partial \chi}{\partial r_{j}}=-\frac{1}{8}(D-1)^{2} \sum_{j=1}^{N-1} \frac{1}{r_{j}} \frac{\partial \log \omega}{\partial r_{j}} .
$$

To calculate the second term we use the law of cosines in the form

$$
g_{i ; j k}=\frac{r_{i j}^{2}+r_{i k}^{2}-r_{j k}^{2}}{2 r_{i j} r_{i k}},
$$

which yields

$$
\frac{\partial g_{i ; j k}}{\partial r_{i j}}=\frac{1}{r_{i k}}-\frac{1}{r_{i j}} g_{i ; j k} .
$$

By repeated use of Eq. (A14) we find 


$$
\begin{aligned}
\sum_{j, k=1}^{N-1} g_{j k} \chi^{-1} \frac{\partial^{2} \chi}{\partial r_{j} \partial r_{k}}= & \chi^{-1} \sum_{j, k=1}^{N-1} \frac{\partial}{\partial r_{j}}\left(g_{j k} \frac{\partial \chi}{\partial r_{k}}\right) \\
& -\chi^{-1} \sum_{j, k=1}^{N-1}\left(\frac{1}{r_{k}}-\frac{1}{r_{j}} g_{j k}\right) \frac{\partial \chi}{\partial r_{k}} \\
= & -\frac{1}{8}(N-1)(D-1) \sum_{j=1}^{N-1} \frac{1}{r_{j}} \frac{\partial \log \omega}{\partial r_{j}}
\end{aligned}
$$

${ }^{1}$ M. Moodley, Ph.D. thesis, University of Rhode Island, 2003.

${ }^{2}$ X.-Y. Gu, Z.-Q. Ma, and J.-Q. Sun, Europhys. Lett. 5, 586 (2003).

${ }^{3}$ V. A. Fock, Bull. Acad. Sci USSR, Phys. Ser., 2, 169 (1935).

${ }^{4} \mathrm{~J}$. Avery, Hyperspherical Harmonics: Applications in Quantum Theory (Kluwer Academic, Boston, 1989).

${ }^{5}$ D. R. Herrick, J. Math. Phys. 16, 281 (1975).

${ }^{6}$ D. R. Herrick and F. H. Stillinger, Phys. Rev. A 11, 42 (1975).

${ }^{7}$ M. P. Nightingale and V. Melik-Alaverdian, Phys. Rev. Lett. 87, 043401 (2001)
${ }^{8}$ M. P. Nightingale and V. Melik-Alaverdian, in Recent Advances in Quantum Monte Carlo Methods, Recent Advances in Computational Chemistry Vol. No. 2, edited by W. A. Lester, Jr., S. M. Rothstein, and S. Tanaka (World Scientific, Singapore, 2002), Pt. II.

${ }^{9}$ J. de Boer, Physica (Amsterdam) 14, 139 (1948).

${ }^{10}$ M. P. Nightingale (unpublished).

${ }^{11}$ D. M. Ceperley and B. Bernu, J. Chem. Phys. 89, 6316 (1988).

${ }^{12}$ B. Bernu, D. M. Ceperley, and W. A. Lester, Jr., J. Chem. Phys. 93, 552 (1990)

${ }^{13}$ W. R. Brown, W. A. Glauser, and W. A. Lester, Jr., J. Chem. Phys. 103, 9721 (1995)

${ }^{14}$ M. Meierovich, A. Mushinski, and M. P. Nightingale, J. Chem. Phys. 105, 6498 (1996)

${ }^{15}$ Our treatment is related to the one given in Ref. 16. In comparison with their Sec. III, it should be noted that our coordinate transformation from Cartesian coordinates to interparticle distance has no inverse; nor do theirs, for that matter.

${ }^{16}$ J. Avery, D. G. Goodson, and D. R. Herschbach, Theor. Chim. Acta 81, 1 (1991).

${ }^{17}$ Q. Shi, S. Kais, and D. R. Herschbach, J. Chem. Phys. 120, 2199 (2004) and references therein.

${ }^{18}$ A. Gonzalez and D. Leal, J. Phys. B 26, 1253 (1993). 